Article

\title{
Fundamental Definitions for Axially-Strained Piezo-Semiconductive Nanostructures
}

\author{
Peyman Amiri (D) and Christian Falconi *
}

check for

updates

Citation: Amiri, P.; Falconi, C. Fundamental Definitions for Axially-Strained Piezo-Semiconductive Nanostructures. Micromachines 2021, 12, 20. https://dx.doi.org/10.3390/ mi12010020

Received: 30 November 2020 Accepted: 24 December 2020 Published: 27 December 2020

Publisher's Note: MDPI stays neutral with regard to jurisdictional claims in published maps and institutional affiliations.

Copyright: () 2020 by the authors. Licensee MDPI, Basel, Switzerland. This article is an open access article distributed under the terms and conditions of the Creative Commons Attribution (CC BY) license (https: / / creativecommons.org/ licenses/by/4.0/).
Department of Electronic Engineering, University of Rome Tor Vergata, 00133 Roma, Italy; peymanamiri1992@gmail.com

* Correspondence: falconi@eln.uniroma2.it

\begin{abstract}
Piezoelectric nanotransducers may offer key advantages in comparison with conventional piezoelectrics, including more choices for types of mechanical input, positions of the contacts, dimensionalities and shapes. However, since most piezoelectric nanostructures are also semiconductive, modeling becomes significantly more intricate and, therefore, the effects of free charges have been considered only in a few studies. Moreover, the available reports are complicated by the absence of proper nomenclature and figures of merit. Besides, some of the previous analyses are incomplete. For instance, the local piezopotential and free charges within axially strained conical piezo-semiconductive nanowires have only been systematically investigated for very low doping $\left(10^{16} \mathrm{~cm}^{-3}\right)$ and under compression. Here we give the definitions for the enhancement, depletion, base and tip piezopotentials, their characteristic lengths and both the tip-to-base and the depletion-toenhancement piezopotential-ratios. As an example, we use these definitions for analyzing the local piezopotential and free charges in n-type $\mathrm{ZnO}$ truncated conical nanostructures with different doping levels (intrinsic, $10^{16} \mathrm{~cm}^{-3}, 10^{17} \mathrm{~cm}^{-3}$ ) for both axial compression and traction. The definitions and concepts presented here may offer insight for designing high performance piezosemiconductive nanotransducers.
\end{abstract}

Keywords: piezoelectric nanotransducers; depletion piezopotential; enhancement piezopotential; base piezopotential; tip piezopotential; characteristic lengths of piezopotentials; depletionto-enhancement piezopotential ratio; tip-to-base piezopotential ratio; piezoelectric nanogenerators; piezotronics

\section{Introduction}

Piezoelectric nanotransducers [1] may offer key advantages with respect to conventional piezoelectric devices and their potential has already been demonstrated in a wide variety of applications, including strain [2] and acceleration sensing [3], measurement of cellular deformations as small as $1 \mathrm{~nm}$ [4], human-motion harvesting [5,6], implantable devices for energy harvesting [7-10], optoelectronics [11], piezocatalysis [12,13], gas detection [14], temperature sensing [15,16], wireless transducers for converting ultrasounds into electrical stimulations [17-20], and more. In particular, in comparison with traditional piezoelectric transducers, at nanoscale there are more degrees of freedom as there can be more options for the types of mechanical input [1,21], the positions of the contacts [1,21], the dimensionalities [1,21], and the shapes [1,21-24]. Despite these and other [1] opportunities, nanoscale dimensions typically result in complications associated to fabricating, characterizing and packaging that make simulations possibly even more important than for conventional devices [25,26]. After the first report on piezoelectric nanogenerators [27], the electric potential within a $\mathrm{ZnO}$ nanowire laterally bent by the tip of an atomic force microscope was computed [28]. Subsequently, simulations showed that this configuration is not ideal for energy harvesting because the region under maximum strain is somehow screened by the conductive seed layer [21] and other types of mechanical input, positions of contacts and shapes were systematically investigated [21], including vertical 
compression which was numerically found to be superior for energy harvesting. Though these simulations offered precious insight for design and led to conclusions confirmed by many experiments, for simplicity, free charges were not taken into account, which is generally a crude approximation as in most cases piezoelectric nanotransducers are made of materials which are both piezoelectric and semiconductive. For instance, in contrast with many other piezoelectric and insulating materials, $\mathrm{ZnO}$ nanostructures can be synthesized with low-temperature, low-cost solution methods $[29,30]$ on practically every substrate, including flexible and stretchable substrates or even conventional printed circuit boards [31]. $\mathrm{ZnO}$ is a semiconductor and even in absence of intentional doping may not be considered as insulating or even intrinsic as $\mathrm{ZnO}$ nanostructures typically exhibit a natural n-type behavior, likely due to non-perfect stoichiometry native defects such as oxygen vacancies or interstitial zinc atoms or hydrogen [32]. As a result, though more simplified simulations can still be useful and provide preliminary information [21,24,28,33], for accurate analyses free charges must be considered [22,23,33-36]. In fact, cylindrical $\mathrm{ZnO}$ nanowires have been simulated with taking into account the presence of free charges both in case of lateral bending [33] and vertical compression [34]. Similarly, the piezopotential in vertically compressed [22,23] or laterally bent [23] truncated conical ZnO nanowires has been studied for different doping levels. However, in these early works, the discussion is complicated by the absence of proper nomenclature and figures of merit. Important cases have not been considered. Here we give fundamental definitions for enabling more accurate and more systematic analyses of axially-strained piezo-semiconductive nanowires. As an important example, we apply these definitions to axially strained truncated conical nanowires. In fact, the local piezopotential and free charges within axially strained conical piezo-semiconductive nanowires have only been investigated for very low doping $\left(10^{16} \mathrm{~cm}^{-3}\right)$ and only under compression [22]. In fact, though, at least for the case of compression, the potential difference between the tip and the base has also been computed for different doping levels [23], both the local electrical potential and free charges have not been reported (for both compression and traction). We compute the local piezopotentials as well as the carrier concentrations along the nanostructure both in case of traction and compression for $2 \mu \mathrm{m}$ long $\mathrm{ZnO}$ truncated conical nanowires with different doping levels (intrinsic, $10^{16} \mathrm{~cm}^{-3}$ and $10^{17} \mathrm{~cm}^{-3}$ ), base radius equal to $150 \mathrm{~nm}$ and radius of the tip ranging from 25 to $125 \mathrm{~nm}$.

\section{Materials and Methods}

For simulations, similar to previous studies [22,23,28,33,34,36] we consider $\mathrm{ZnO}$ nanostructures because of their remarkable practical advantages, including piezoelectricity and easy fabrication with low-temperature, low-cost, large-area wet-chemical methods which are suitable for almost every substrate, including CMOS devices or plastic and flexible substrates. We used the $\mathrm{ZnO}$ conical nanowires and boundary conditions schematically shown in Figure 1. The nanowire length is $2 \mu \mathrm{m}$ and the bottom radius is $150 \mathrm{~nm}$. The tip radius is smaller than the bottom radius in order to form a truncated conical structure and has been changed from 25 to $125 \mathrm{~nm}$. Axial compression or traction forces equal to $442 \mathrm{nN}$ have been uniformly applied $[22,34]$ to the nanowire tip and both the electric potential and the free charges concentrations have been calculated. At the bottom of the nanowire we applied a mechanical constrain $(u=0)$ and the tapered tip is only free to move axially. At the center of the nanowire the electric potential is fixed to $\mathrm{V}=0$ in order to not perturb the piezopotential developed at the bottom of the nanowire [22]. The nanowire is surrounded by air. The material properties for $\mathrm{ZnO}$ are given in Table 1. 


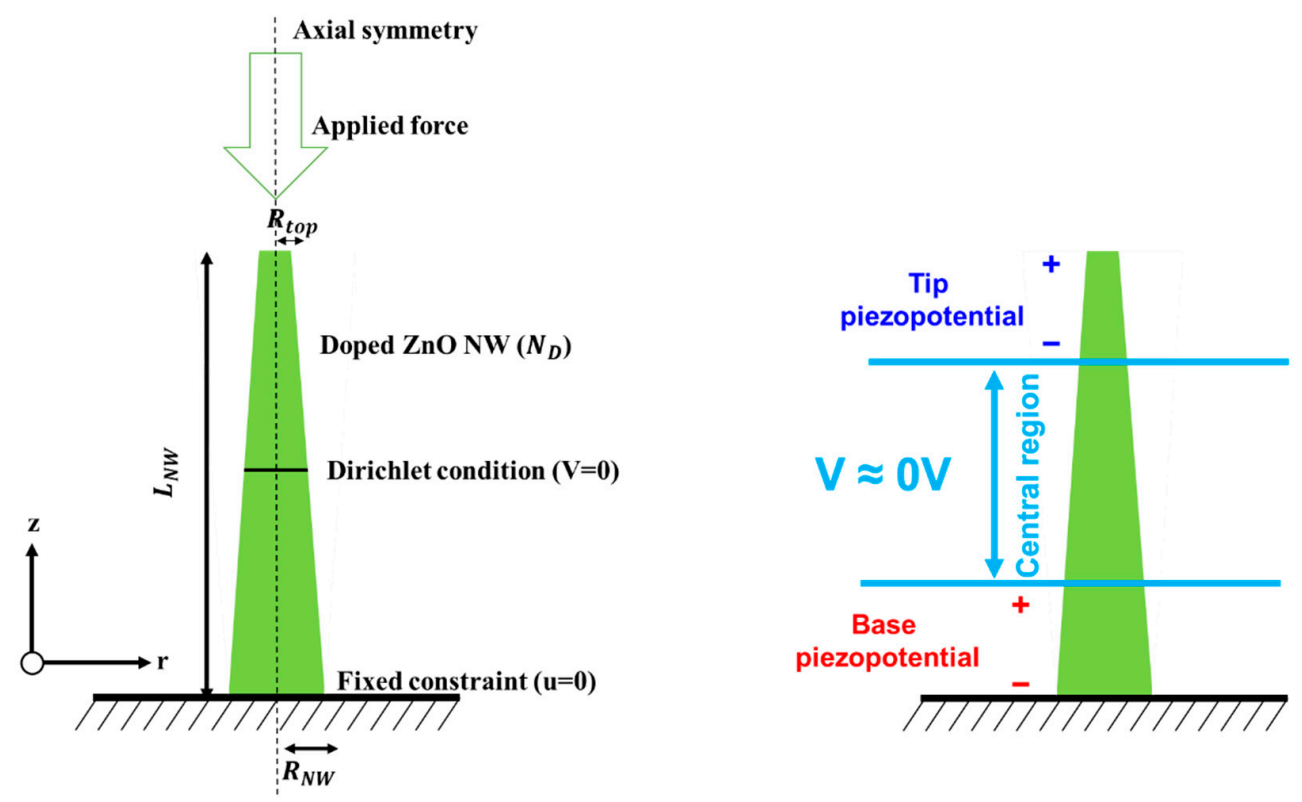

Figure 1. Schematic representations of the truncated conical nanowires illustrating (left) the boundary conditions and the coordinate system, with definitions of $L_{N W}$ (length), $R_{N W}$ (base radius), $R_{T I P}$ (tip radius) and (right) the tip piezopotential, the base piezopotential and the central region with an almost zero potential.

Table 1. Properties of the $\mathrm{ZnO}$ nanowires used in the simulations.

\begin{tabular}{ccc}
\hline Young's Modulus & Poisson's Ratio & Piezoelectric Coefficients (e33) \\
\hline $129 \mathrm{GPa}$ & $\mathrm{v}=0.349$ & $1.22 \mathrm{C} \mathrm{m}^{-2}$ \\
\hline
\end{tabular}

The COMSOL software has been used to simulate the nanowire. The geometry has been created using the 2D axisymmetric method. The zero-displacement condition has been applied to the bottom of the nanowire while the top tip is free to move and will experience either compression or traction force. The Dirichlet condition $(\mathrm{V}=0)$ is applied at the center of the nanowire. In order to consider the effect of charge carriers, the equations for semiconductors have been added to the simulations and, similar to [22,23,34], for simplicity, the effects of external charges [37-39] have not been considered. The nanowire is assumed to be uniformly n-type doped, with different doping conditions (intrinsic, $\left.10^{16} \mathrm{~cm}^{-3}, 10^{17} \mathrm{~cm}^{-3}\right)$.

\section{Results}

\subsection{Depletion Piezopotential $\left(\Delta V_{P Z-D E P L}\right)$ and Enhancement Piezopotential ( $\left.\triangle V_{P Z-E N H A N C}\right)$}

The application of strain to a piezo-semiconductive nanostructure will result, in comparison with the initial, unstrained nanostructure, in both enhancement and depletion regions where the free charges will be increased or reduced, respectively [22,23,33,34]. In case of vertical compression or traction applied to axial nanostructures with a constant doping, if, for symmetry, the base is left floating [22], there will be only one depletion region and one enhancement region, at the tip or at the base of the nanowire, depending on the type ( $p$ or $n$ ) of doping and on the sign of the force (traction or compression) [22,34]. If the length of the nanowire is sufficient, the depletion and the enhancement regions will have no overlaps. Each region will have a sharp limit (tip or base) and an asymptotic limit at the center of the nanowire. In such conditions we define the depletion piezopotential, $\triangle V_{P Z-D E P L}$, and the enhancement piezopotential, $\triangle V_{P Z-E N H A N C}$, as the potential drops in the depletion and in the enhancement regions, respectively. For consistency with literature, both these potentials must be conventionally taken as the difference between the extremity of the (depletion or enhancement, respectively) region closer to the tip and the extremity 
closer to the base. With these definitions, the total voltage drop between the tip and the base is the sum of the depletion piezopotential and of the enhancement piezopotential. Since we ignore other effects (e.g., the band bending due to the metal-semiconductor interface) and only consider the piezopotentials due to strain, the depletion piezopotential and the enhancement piezopotential will always be concordant. Though the total potential drop already provides important information, in many cases and, for instance, when designing piezotronic devices, both the depletion piezopotential and the enhancement piezopotential are crucial as the charge transport properties at a certain junction will depend on the correspondent local piezopotential.

\subsection{Tip Piezopotential $\left(\Delta V_{P Z-T I P}\right)$ and Base Piezopotential $\left(\Delta V_{P Z-B A S E}\right)$}

In the analysis of conical nanowires, it is also useful to define the tip piezopotential, $\triangle V_{P Z-T I P}$, and the base piezopotential, $\Delta V_{P Z-B A S E}$, as the potential drops developed at the tip and base, respectively. Similar to the depletion piezopotential and the enhancement piezopotential, as schematically shown in Figure 1, the tip piezopotential and the base piezopotential will also be conventionally taken as the difference between the tip and the center or between the center and the base, respectively. Clearly, depending on the sign of the axial force and on the type of doping, the base and the tip will be depleted and enhanced, respectively, or vice versa. In cylindrical nanowires, the base and tip have the same radius, but in tapered nanowires the tip area may be much smaller than the base area, which may translate in higher strains which will tend to result in higher piezopotentials at the tip.

\subsection{Characteristic Lengths of the Piezopotentials (Tip, Base, Depletion or Enhancement Piezopotential)}

In the analysis of axially strained nanowires it is useful to quantitatively evaluate the width of the enhancement or depletion regions. However, since the charge concentrations can have complex profiles, instead of considering the charges, we may consider the piezopotentials and define, for each piezopotential (tip, base, depletion or enhancement piezopotential), its characteristic length as the width of the region, starting from the extremity (tip or base) and going toward the center, where most of the correspondent piezopotential drop occurs (e.g., for our simulations we will consider $90 \%$ of the piezopotential drop).

\subsection{Depletion-to-Enhancement Piezopotential Ratio ( $\left.r_{P Z, D E P L-T O-E N H A N C}\right)$}

Since free charges tend to screen the piezopotential, the depletion piezopotential tends to be higher than the enhancement piezopotential. The superior ability of the depletion region to develop a piezopotential when strained may be quantified by the depletion-toenhancement piezopotential ratio, $r_{P Z, D E P L-T O-E N H A N C}$, defined as the ratio between the depletion piezopotential and the enhancement piezopotential. Since both the depletion piezopotential and the enhancement piezopotential are always concord, $r_{P Z, D E P L-T O-E N H A N C}$ is always positive.

\subsection{Tip-to-Base Piezopotential Ratio ( $\left.r_{P Z, T I P-T O-B A S E}\right)$}

In cylindrical nanowires the base area and the tip area are identical and, therefore, for a given axial input force, the strain is homogeneous along the entire nanostructure. By contrast, for piezo-semiconductive nanostructures with non-constant cross sections [22,23], for a given input force, different regions are subject to different strains. As a consequence, one region, subject to higher strain, may tend to have a higher magnitude of the piezopotential. The ability of the tip to develop an higher piezopotential may be quantified by the tip-tobase piezopotential ratio, $r_{P Z, T I P-T O-B A S E}$, defined as the ratio between the tip piezopotential and the base piezopotential. Since the tip piezopotential and the base piezopotential are always concord, $r_{P Z, T I P-T O-B A S E}$ is always positive. 


\subsection{Piezopotential in Truncated Conical Dielectric Nanowires under Vertical Compression or Traction}

Figure 2 shows the piezoelectric potential within vertically compressed conical truncated dielectric nanowires. As evident, the local piezopotential at the base of the nanowire is almost unaffected by the change of the tip radius. In fact, for a given compression force, since the base area is constant, the pressure exerted on the base of the nanowire due to the mechanical boundary condition at the base (i.e., no displacement at the base) does not depend on the tip radius. As a consequence, the strain at the base and, therefore, the piezopotential at the base are almost perfectly independent on the tip radius. As expected, when the tip radius is reduced, the higher strain at the tip will result in higher electric field and potential drops.

\section{Compression (442 nN) - Dielectric}

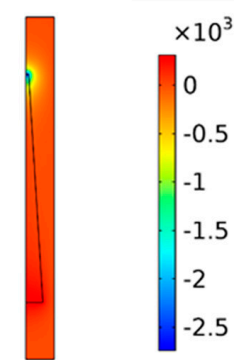

(a)

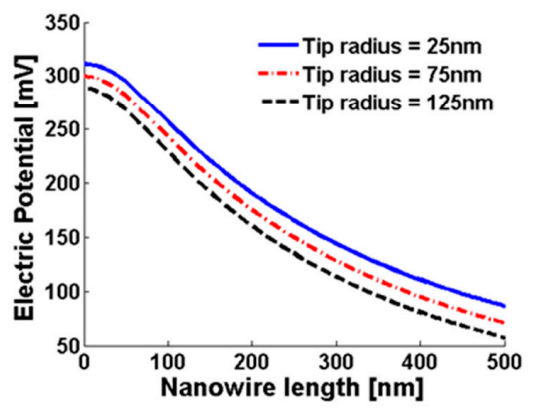

(d)

\section{Electric Potential [mV]}

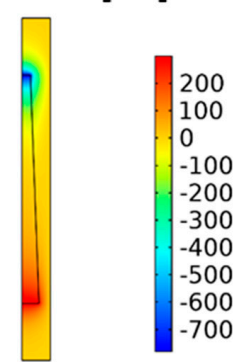

(b)

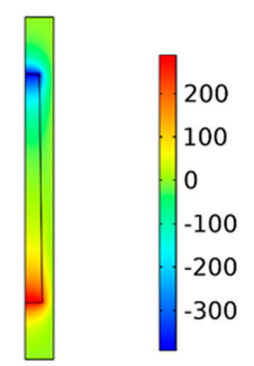

(c)

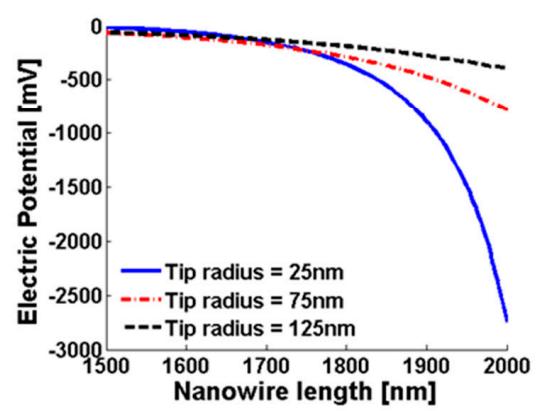

(e)

Figure 2. Piezoelectric potential within conical truncated dielectric nanowires with length $2 \mu \mathrm{m}$, base radius $150 \mathrm{~nm}$ and compressive force $442 \mathrm{nN}$. (a-c) Color maps of the piezopotential for tip radius equal to $125 \mathrm{~nm}(\mathbf{a}), 75 \mathrm{~nm}$ (b) and $25 \mathrm{~nm}$ (c). (d,e) Base piezopotential (d) and tip piezopotential (e) for different values of the tip radius.

Figure 3 shows similar results for the case of traction. As expected, the sign of the piezopotentials are opposite, but the magnitude of the tip piezopotential is still larger in comparison with the base piezopotential due to the smaller area and, therefore, higher strain at the tip. 


\section{Traction (442 nN) - Dielectric}

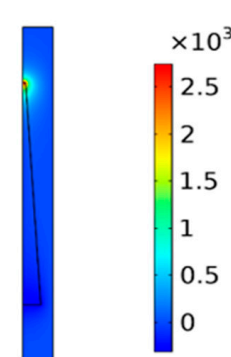

(a)

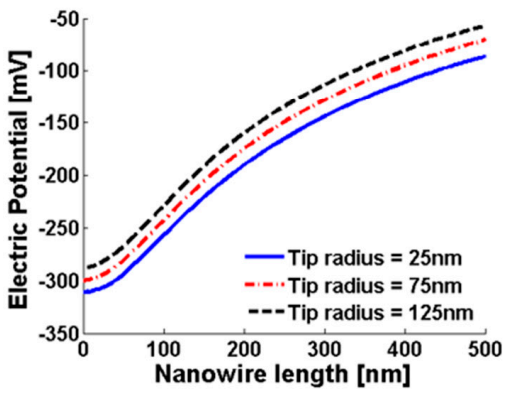

(d)

\section{Electric Potential [mV]}

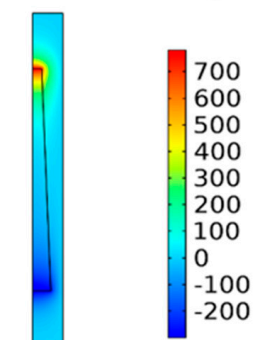

(b)

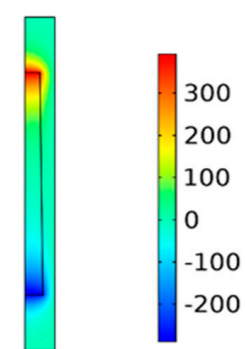

(c)

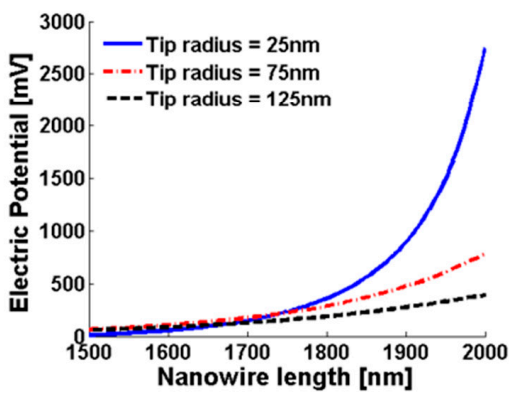

(e)

Figure 3. Piezoelectric potential within conical truncated dielectric nanowires with length $2 \mu \mathrm{m}$, base radius $150 \mathrm{~nm}$ and traction force $442 \mathrm{nN}$. (a-c) Color maps of the piezopotential for tip radius equal to $125 \mathrm{~nm}(\mathbf{a}), 75 \mathrm{~nm}$ (b) and $25 \mathrm{~nm}$ (c). (d,e) Base piezopotential (d) and tip piezopotential (e) for different values of the tip radius.

3.7. Piezopotentials, Free Carrier Concentrations, Piezopotential Ratios and Characteristic Lengths of the Piezopotentials in Truncated Conical Nanowires with $10^{16} \mathrm{~cm}^{-3}$ Doping under Vertical Compression or Traction

Figures 4 and 5 show the piezoelectric potential and the free carrier concentrations, respectively, within vertically compressed $\mathrm{ZnO}$ truncated conical nanowires with $10^{16} \mathrm{~cm}^{-3}$ doping.

Figures 6 and 7 show the piezoelectric potential and the free carrier concentrations, respectively, within vertically pulled $\mathrm{ZnO}$ truncated conical nanowires with $10^{16} \mathrm{~cm}^{-3}$ doping.

Tables 2 and 3 show the piezopotentials, the piezopotentials-ratios and the characteristic lengths of the piezopotentials for $2 \mu \mathrm{m}$ long $\mathrm{n}$-type $\mathrm{ZnO}$ truncated conical nanowires with $10^{16} \mathrm{~cm}^{-3}$ doping under axial compression (Table 2) and traction (Table 3).

Table 2. Piezopotentials, piezopotentials-ratios and characteristic lengths of the piezopotentials for $2 \mu \mathrm{m}$ long n-type $\mathrm{ZnO}$ truncated conical nanowires with $150 \mathrm{~nm}$ base radius and $10^{16} \mathrm{~cm}^{-3}$ doping under axial compression.

\begin{tabular}{|c|c|c|c|}
\hline \multicolumn{4}{|c|}{$N_{D}=10^{16} \mathrm{~cm}^{-3}$, Compression $(442 \mathrm{nN})$} \\
\hline$R_{T I P}$ & $25 \mathrm{~nm}$ & $75 \mathrm{~nm}$ & $125 \mathrm{~nm}$ \\
\hline$\Delta V_{P Z-D E P L}=\Delta V_{P Z-T I P}$ & $-1540 \mathrm{mV}$ & $-180 \mathrm{mV}$ & $-52 \mathrm{mV}$ \\
\hline$\Delta V_{P Z-E N H A N C}=\Delta V_{P Z-B A S E}$ & $-21 \mathrm{mV}$ & $-20 \mathrm{mV}$ & $-19 \mathrm{mV}$ \\
\hline$r_{P Z, D E P L-T O-E N H A N C}=r_{P Z, T I P-T O-B A S E}$ & 73 & 9 & 2.7 \\
\hline Characteristic length $\left(\Delta V_{P Z-D E P L}=\Delta V_{P Z-T I P}\right)$ & $165 \mathrm{~nm}$ & $115 \mathrm{~nm}$ & $94 \mathrm{~nm}$ \\
\hline Characteristic length $\left(\Delta V_{P Z-E N H A N C}=\Delta V_{P Z-B A S E}\right)$ & $83 \mathrm{~nm}$ & $80 \mathrm{~nm}$ & $80 \mathrm{~nm}$ \\
\hline
\end{tabular}




\section{Compression (442 nN) - doping 1 e16}

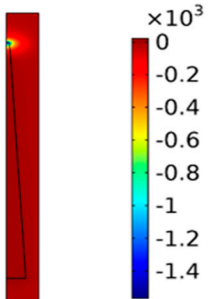

(a)

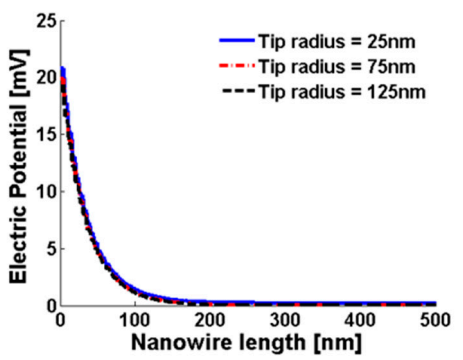

(d)

\section{Electric Potential [mV]}

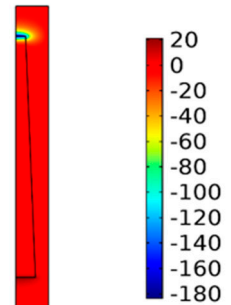

(b)

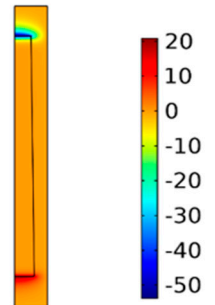

(c)

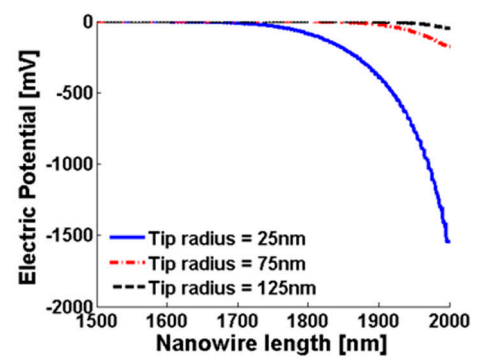

(e)

Figure 4. Piezoelectric potential within conical truncated nanowires with length $2 \mu \mathrm{m}$, base radius $150 \mathrm{~nm}$, compressive force $442 \mathrm{nN}$ and n-type $10^{16} \mathrm{~cm}^{-3}$ doping. (a-c) Color maps of the piezopotential for tip radius equal to $125 \mathrm{~nm}(\mathbf{a}), 75 \mathrm{~nm}(\mathbf{b})$ and $25 \mathrm{~nm}$ (c). (d,e) Base (enhancement) piezopotential (d) and tip (depletion) piezopotential (e) for different values of the tip radius.

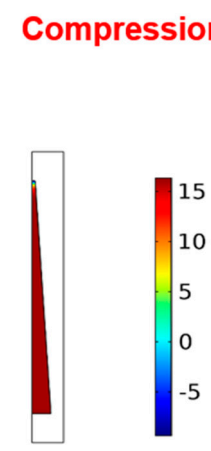

(a)

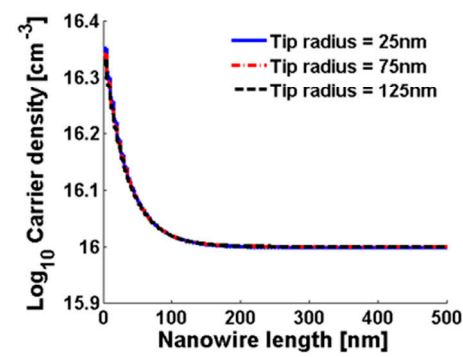

(d) $\log _{10}$ Carrier density $\left(\mathrm{cm}^{-3}\right)$

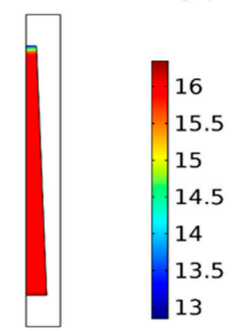

(b)

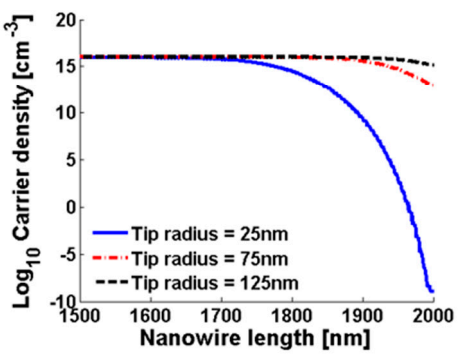

(e)

Figure 5. Free charge concentrations within conical truncated nanowires with length $2 \mu \mathrm{m}$, base radius $150 \mathrm{~nm}$, compressive force $442 \mathrm{nN}$ and n-type $10^{16} \mathrm{~cm}^{-3}$ doping. (a-c) Color maps of the free charge concentrations for tip radius equal to $125 \mathrm{~nm}$ (a), $75 \mathrm{~nm}$ (b) and $25 \mathrm{~nm}$ (c). (d,e) Charge concentration at the base (enhancement) (d) and tip (depletion) (e) for different values of the tip radius. 


\section{Traction (442 $\mathrm{nN})$ - doping $1 \mathrm{e} 16$}

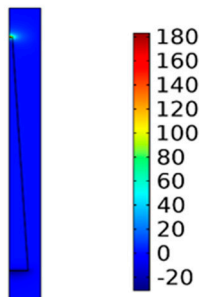

(a)

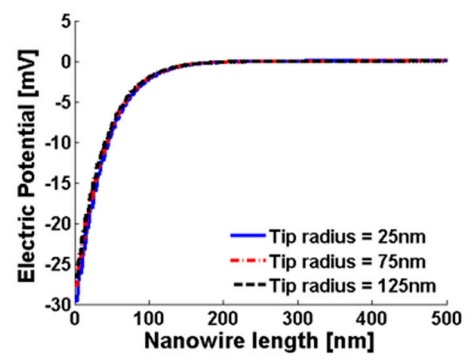

(d)

\section{Electric Potential [mV]}

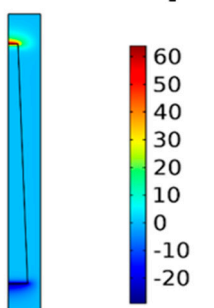

(b)

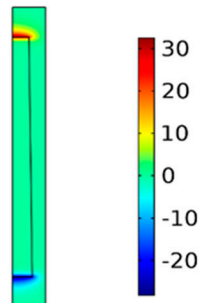

(c)

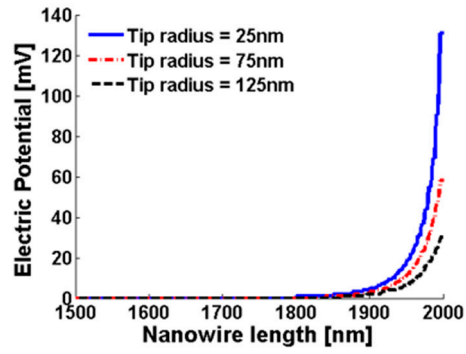

(e)

Figure 6. Piezoelectric potential within conical truncated nanowires with length $2 \mu \mathrm{m}$, base radius $150 \mathrm{~nm}$, traction force $442 \mathrm{nN}$ and n-type $10^{16} \mathrm{~cm}^{-3}$ doping. (a-c) Color maps of the piezopotential for tip radius equal to $125 \mathrm{~nm}(\mathbf{a}), 75 \mathrm{~nm}$ (b) and $25 \mathrm{~nm}$ (c). (d,e) Base (enhancement) piezopotential (d) and tip (depletion) piezopotential (e) for different values of the tip radius.

\section{Traction (442 $\mathrm{nN})$ - doping $1 \mathrm{e} 16$}

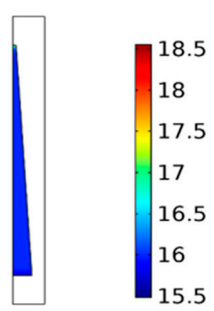

(a)

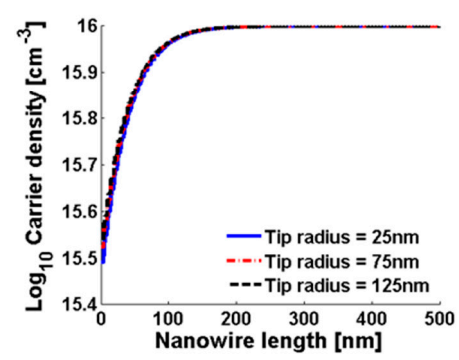

(d)

\section{$\log _{10}$ Carrier density $\left(\mathrm{cm}^{-3}\right)$}

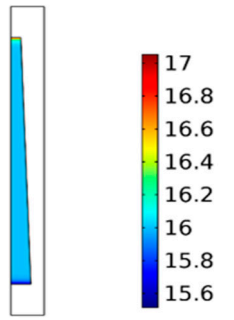

(b)

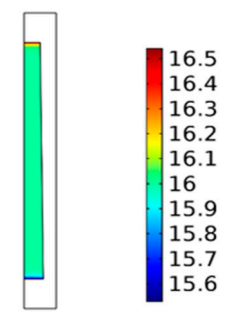

(c)

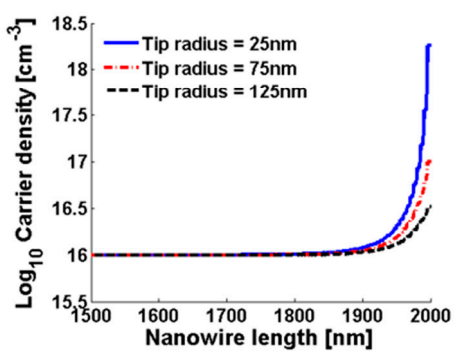

(e)

Figure 7. Free charge concentrations within conical truncated nanowires with length $2 \mu \mathrm{m}$, base radius $150 \mathrm{~nm}$, traction force $442 \mathrm{nN}$ and n-type $10^{16} \mathrm{~cm}^{-3}$ doping. (a-c) Color maps of the free charge concentrations for tip radius equal to $125 \mathrm{~nm}(\mathbf{a}), 75 \mathrm{~nm}$ (b) and $25 \mathrm{~nm}$ (c). (d,e) Charge concentration at the base (enhancement) (d) and tip (depletion) (e) for different values of the tip radius. 
Table 3. Piezopotentials, piezopotentials-ratios and characteristic lengths of the piezopotentials for $2 \mu \mathrm{m}$ long n-type $\mathrm{ZnO}$ truncated conical nanowires with $150 \mathrm{~nm}$ base radius and $10^{16} \mathrm{~cm}^{-3}$ doping under axial traction.

\begin{tabular}{cccc}
\hline \multicolumn{4}{c}{$\boldsymbol{N}_{\boldsymbol{D}}=\mathbf{1 0}^{\mathbf{1 6}} \mathbf{c m}^{\mathbf{- 3}}$, Traction $\left.\mathbf{( 4 4 2} \mathbf{~ n N}\right)$} \\
$R_{T I P}$ & $25 \mathrm{~nm}$ & $75 \mathrm{~nm}$ & $125 \mathrm{~nm}$ \\
$\Delta V_{P Z-D E P L}=\Delta V_{P Z-B A S E}$ & $131 \mathrm{mV}$ & $58 \mathrm{mV}$ & $30 \mathrm{mV}$ \\
$\Delta V_{P Z-E N H A N C}=\Delta V_{P Z-T I P}$ & $30 \mathrm{mV}$ & $28 \mathrm{mV}$ & $27 \mathrm{mV}$ \\
$r_{P Z, D E P L-T O-E N H A N C}=1 / r_{P Z, T I P-T O-B A S E}$ & 4.4 & 2.1 & 1.1 \\
$r_{P Z, T I P-T O-B A S E}=1 / r_{P Z, D E P L-T O-E N H A N C}$ & 0.23 & 0.48 & 0.9 \\
Characteristic length $\left(\Delta V_{P Z-E N H A N C}=\Delta V_{P Z-T I P}\right)$ & $60 \mathrm{~nm}$ & $74 \mathrm{~nm}$ & $79 \mathrm{~nm}$ \\
Characteristic length $\left(\Delta V_{P Z-D E P L}=\Delta V_{P Z-B A S E}\right)$ & $90 \mathrm{~nm}$ & $89 \mathrm{~nm}$ & $89 \mathrm{~nm}$ \\
\hline
\end{tabular}

3.8. Piezopotentials, Free Carrier Concentrations, Piezopotential Ratios and Characteristic Lengths of the Piezopotentials in Truncated Conical Nanowires with $10^{17} \mathrm{~cm}^{-3}$ Doping under Vertical Compression or Traction

Figures 8 and 9 show the piezoelectric potential and the free carrier concentrations, respectively, within vertically compressed $\mathrm{ZnO}$ truncated conical nanowires with $10^{17} \mathrm{~cm}^{-3}$ doping.

\section{Compression (442 nN) - doping 1 e17}

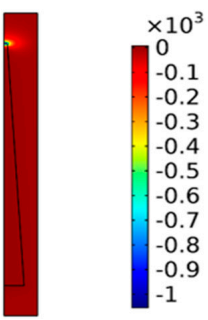

(a)

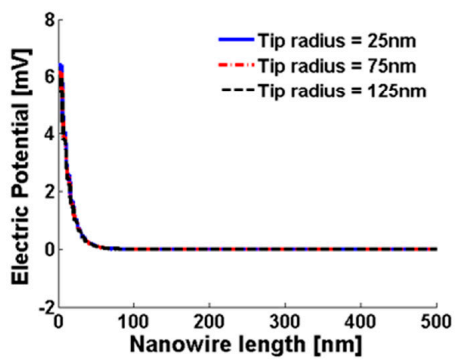

(d)

\section{Electric Potential [mV]}

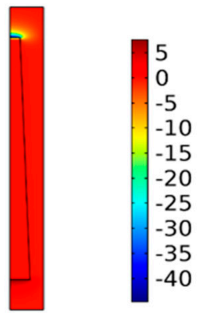

(b)

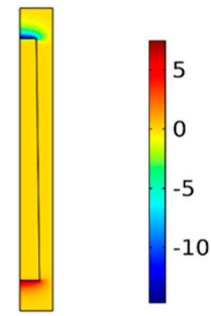

(c)

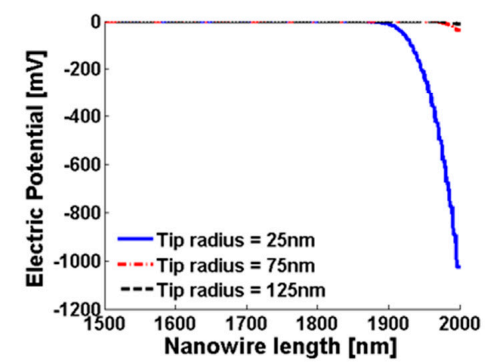

(e)

Figure 8. Piezoelectric potential within conical truncated nanowires with length $2 \mu \mathrm{m}$, base radius $150 \mathrm{~nm}$, compressive force $442 \mathrm{nN}$ and n-type $10^{17} \mathrm{~cm}^{-3}$ doping. (a-c) Color maps of the piezopotential for tip radius equal to $125 \mathrm{~nm}$ (a), $75 \mathrm{~nm}$ (b) and $25 \mathrm{~nm}$ (c). (d,e) Base (enhancement) piezopotential (d) and tip (depletion) piezopotential (e) for different values of the tip radius.

Figures 10 and 11 show the piezoelectric potential and the free carrier concentrations, respectively, within vertically pulled $\mathrm{ZnO}$ truncated conical nanowires with $10^{17} \mathrm{~cm}^{-3}$ doping. 


\section{Compression (442 nN) - doping 1e17}

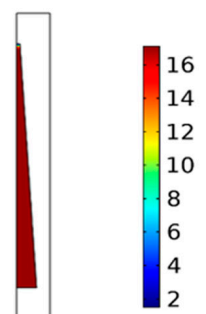

(a)

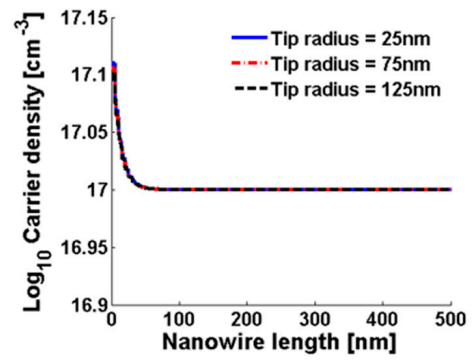

(d) $\log _{10}$ Carrier density $\left(\mathrm{cm}^{-3}\right)$

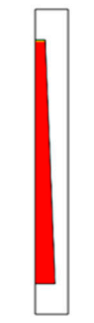

17.1
17
16.9
16.8
16.7
16.6
16.5
16.4
16.3

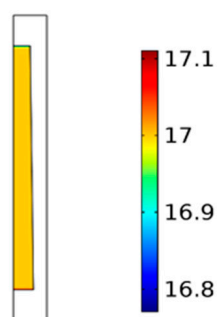

(c)

(b)

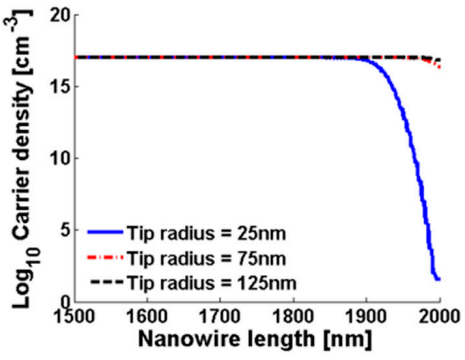

(e)

Figure 9. Free charge concentrations within conical truncated nanowires with length $2 \mu \mathrm{m}$, base radius $150 \mathrm{~nm}$, compressive force $442 \mathrm{nN}$ and n-type $10^{17} \mathrm{~cm}^{-3}$ doping. (a-c) Color maps of the free charge concentrations for tip radius equal to $125 \mathrm{~nm}(\mathbf{a}), 75 \mathrm{~nm}(\mathbf{b})$ and $25 \mathrm{~nm}(\mathbf{c})$. (d,e) Charge concentration at the base (enhancement) (d) and tip (depletion) (e) for different values of the tip radius.

\section{Traction (442 nN) - doping 1e17}

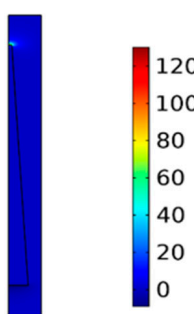

(a)

\section{Electric Potential [mV]}

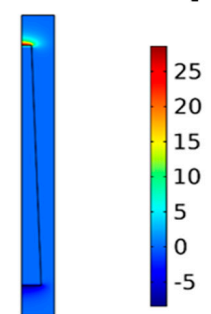

(b)

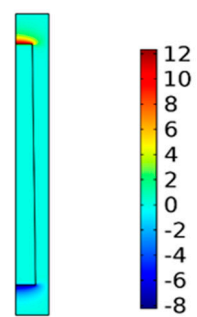

(c)

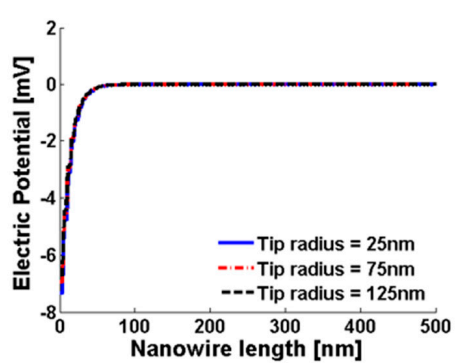

(d)

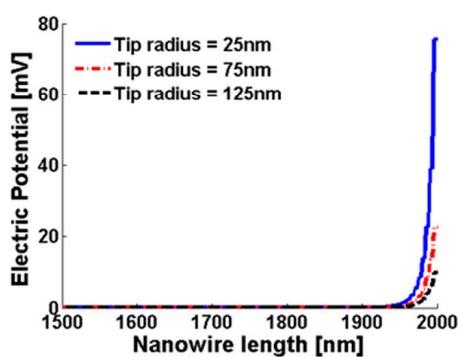

(e)

Figure 10. Piezoelectric potential within conical truncated nanowires with length $2 \mu \mathrm{m}$, base radius $150 \mathrm{~nm}$, traction force $442 \mathrm{nN}$ and n-type $10^{17} \mathrm{~cm}^{-3}$ doping. (a-c) Color maps of the piezopotential for tip radius equal to $125 \mathrm{~nm}$ (a), $75 \mathrm{~nm}$ (b) and $25 \mathrm{~nm}$ (c). (d,e) Base (enhancement) piezopotential (d) and tip (depletion) piezopotential (e) for different values of the tip radius. 


\section{Traction (442 nN) - doping 1e17}

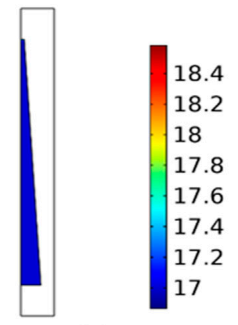

(a)

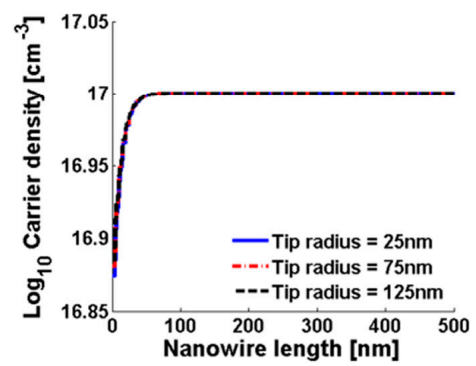

(d) $\log _{10}$ Carrier density $\left(\mathrm{cm}^{-3}\right)$

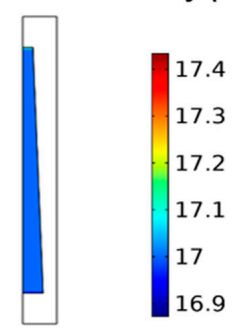

(b)

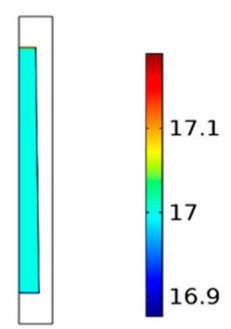

(c)

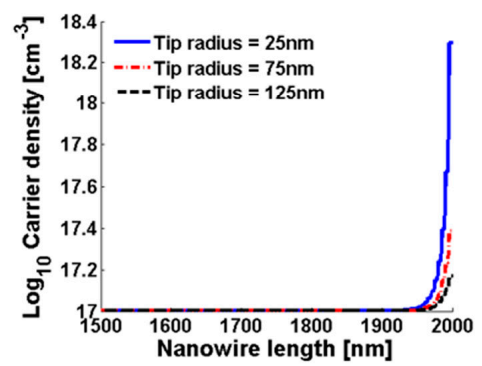

(e)

Figure 11. Free charge concentrations within conical truncated nanowires with length $2 \mu \mathrm{m}$, base radius $150 \mathrm{~nm}$, traction force $442 \mathrm{nN}$ and n-type $10^{17} \mathrm{~cm}^{-3}$ doping. (a-c) Color maps of the free charge concentrations for tip radius equal to $125 \mathrm{~nm}(\mathbf{a}), 75 \mathrm{~nm}$ (b) and $25 \mathrm{~nm}$ (c). (d,e) Charge concentration at the base (enhancement) (d) and tip (depletion) (e) for different values of the tip radius.

Tables 4 and 5 show the piezopotentials, the piezopotentials-ratios and the characteristic lengths of the piezopotentials for $2 \mu \mathrm{m}$ long n-type $\mathrm{ZnO}$ truncated conical nanowires with $10^{17} \mathrm{~cm}^{-3}$ doping under axial compression (Table 4) and traction (Table 5).

Table 4. Piezopotentials and piezopotentials-ratios for $2 \mu \mathrm{m}$ long n-type $\mathrm{ZnO}$ truncated conical nanowires with $150 \mathrm{~nm}$ base radius with $10^{17} \mathrm{~cm}^{-3}$ doping under axial compression.

\begin{tabular}{|c|c|c|c|}
\hline \multicolumn{4}{|c|}{$N_{D}=10^{17} \mathrm{~cm}^{-3}$, Compression $(442 \mathrm{nN})$} \\
\hline$R_{\text {TIP }}$ & $25 \mathrm{~nm}$ & $75 \mathrm{~nm}$ & $125 \mathrm{~nm}$ \\
\hline$\Delta V_{P Z-D E P L}=\Delta V_{P Z-T I P}$ & $-1028 \mathrm{mV}$ & $-38 \mathrm{mV}$ & $-12 \mathrm{mV}$ \\
\hline$\Delta V_{P Z-E N H A N C}=\Delta V_{P Z-B A S E}$ & $-6 \mathrm{mV}$ & $-6 \mathrm{mV}$ & $-6 \mathrm{mV}$ \\
\hline$r_{P Z, D E P L-T O-E N H A N C}=r_{P Z, T I P-T O-B A S E}$ & 170 & 6.3 & 2 \\
\hline Characteristic length $\left(\Delta V_{P Z-D E P L}=\Delta V_{P Z-T I P}\right)$ & $66 \mathrm{~nm}$ & $31 \mathrm{~nm}$ & $30 \mathrm{~nm}$ \\
\hline Characteristic length $\left(\Delta V_{P Z-E N H A N C}=\Delta V_{P Z-B A S E}\right)$ & $29 \mathrm{~nm}$ & $29 \mathrm{~nm}$ & $29 \mathrm{~nm}$ \\
\hline
\end{tabular}

Table 5. Piezopotentials, piezopotentials-ratios and characteristic lengths of the piezopotentials for $2 \mu \mathrm{m}$ long n-type $\mathrm{ZnO}$ truncated conical nanowires with $150 \mathrm{~nm}$ base radius and $10^{17} \mathrm{~cm}^{-3}$ doping under axial traction.

\begin{tabular}{|c|c|c|c|}
\hline \multicolumn{4}{|c|}{$N_{D}=10^{17} \mathrm{~cm}^{-3}$, Traction $(442 \mathrm{nN})$} \\
\hline$R_{T I P}$ & $25 \mathrm{~nm}$ & $75 \mathrm{~nm}$ & $125 \mathrm{~nm}$ \\
\hline$\Delta V_{P Z-D E P L}=\Delta V_{P Z-B A S E}$ & $75 \mathrm{mV}$ & $22 \mathrm{mV}$ & $10 \mathrm{mV}$ \\
\hline$\Delta V_{P Z-E N H A N C}=\Delta V_{P Z-T I P}$ & $7 \mathrm{mV}$ & $7 \mathrm{mV}$ & $7 \mathrm{mV}$ \\
\hline$r_{P Z, D E P L-T O-E N H A N C}=1 / r_{P Z, T I P-T O-B A S E}$ & 10.7 & 3.2 & 1.4 \\
\hline$r_{P Z, T I P-T O-B A S E}=1 / r_{P Z, D E P L-T O-E N H A N C}$ & 0.093 & 0.32 & 0.7 \\
\hline Characteristic length $\left(\Delta V_{P Z-E N H A N C}=\Delta V_{P Z-T I P}\right)$ & $25 \mathrm{~nm}$ & $26 \mathrm{~nm}$ & $29 \mathrm{~nm}$ \\
\hline Characteristic length $\left(\Delta V_{P Z-D E P L}=\Delta V_{P Z-B A S E}\right)$ & $30 \mathrm{~nm}$ & $30 \mathrm{~nm}$ & $30 \mathrm{~nm}$ \\
\hline
\end{tabular}




\section{Discussion and Conclusions}

We have given the fundamental definitions for the analysis of piezosemiconductive nanostructures under axial strain. First, we have defined the depletion piezopotential $\left(\triangle V_{P Z-D E P L}\right)$ and the enhancement piezopotential $\left(\triangle V_{P Z-E N H A N C}\right)$, the tip piezopotential $\left(\Delta V_{P Z-T I P}\right)$ and the base piezopotential $\left(\triangle V_{P Z-B A S E}\right)$. Depending on the type ( $\mathrm{p}$ or $\left.\mathrm{n}\right)$ of doping and on the sign of the force (traction or compression) [22,34] the tip (base) is enhanced (depleted) or depleted (enhanced) and vice versa. All these piezopotentials can be conventionally taken with their positive terminal closer to the tip (i.e., coincident with the tip if the tip is within the region where the piezopotential is or, otherwise, with the center) and the negative terminal closer to the base (i.e., coincident with the base if the base is within the region where the piezopotential is or, otherwise, with the center). These distinct piezopotentials can only be defined if the nanostructure is sufficiently long so that there is region in the center where the piezopotential is almost perfectly constant (i.e., there is almost zero electric field) and the depletion and enhancement regions are clearly distinguishable at the extremities of the nanowires. If the length of the nanowire is insufficient, the depletion and the enhancement regions will overlap and these piezopotentials may not be defined. Clearly, these definitions only apply to semiconductive nanostructures as in dielectric nanowires there is no depletion or enhancement so that it is not possible to define these piezopotentials.

Afterwards, we have proposed to quantitatively evaluate the widths of the regions where the piezopotential develops, instead of referring to the concentrations of free charges, whose total variations strongly depend on the particular case under investigation (e.g., depend on doping level, geometry and applied force), thus not allowing to easily define a figure of merit. With this simplification, for each piezopotential (tip, base, depletion or enhancement piezopotential), its characteristic length can be defined as the width of the region where most of the correspondent piezopotential drop occurs (e.g., for our simulations we will consider $90 \%$ of the piezopotential drop), starting from the extremities (tip or base) and going toward the center.

Other figures of merit which can allow to concisely characterize axially strained piezoelectric nanostructures are the depletion-to-enhancement piezopotential ratio $\left(r_{P Z, D E P L-T O-E N H A N C}\right)$ and the tip-to-base piezopotential ratio $\left(r_{P Z, T I P-T O-B A S E}\right)$ which quantitatively express the asymmetrical ability of the nanostructure to develop the piezopotential in different regions. With our definitions of the fundamental piezopotentials, both these ratios are always positive. As to the expected values for these ratios, the piezopotential is more easily created in depleted regions (rather than in enhanced regions, because of the reduced number of free charges) and at the tip (rather than at the base, because of the reduced area and, therefore, of the higher strains). As a result, when the tip is depleted, these ratios will be identical and will tend to be very high (much higher than 1), whereas if the tip is enhanced these ratios will be reciprocal (multiplicative inverse) and may be larger or smaller than 1.

Finally, as an example, we have applied these definitions to axially strained truncated conical nanowires and computed the local piezopotential and free charges within axially strained conical piezo-semiconductive nanowires both in case of traction and compression for $2 \mu \mathrm{m}$ long $\mathrm{ZnO}$ truncated conical nanowires with different doping levels (insulator, $10^{16} \mathrm{~cm}^{-3}$ and $10^{17} \mathrm{~cm}^{-3}$ ), base radius equal to $150 \mathrm{~nm}$ and radius of the tip ranging from $25 \mathrm{~nm}$ to $125 \mathrm{~nm}$. In these simulations, as expected [22,23,34], the piezopotential tend to be higher in the depletion region and at the tip so that when the depletion region is at the tip (i.e., the enhancement region is at the base), as it is the case for n-type $\mathrm{ZnO}$ nanowires under compression, the ratios $r_{P Z, D E P L-T O-E N H A N C}$ and $r_{P Z, T I P-T O-B A S E}$ are identical and tend to be very high (up to 170), with the highest values found when the tip radius is minimum (i.e., higher pressure and strains at the tip). By contrast, if the depletion region is at the base and the enhancement region is at the tip, as it is the case for n-type $\mathrm{ZnO}$ nanowires under traction, the higher strains at the tip do not necessarily result in significantly higher piezopotentials because of the higher concentrations of free charges at 
the tip. The characteristic lengths of the piezopotentials are always of the same order of magnitude, though, of course, when the depletion region is at the tip and, therefore, under higher strains, the characteristic length of the tip (or depletion) piezopotential may extend significantly more (e.g., up to about 2 times) more than the characteristic length of the base (or enhancement) piezopotential.

Most remarkably, earlier works restricted, at least for some cases (i.e., for some doping level) to general and global quantities, such as the total "tip-to-base" piezopotential drop developed within the nanostructure. Though such an approach already allows to obtain very important information, the charge transport at each junction is controlled by the local piezopotential developed in close proximity of the metal-nanostructure junction and, for this reason, local analyses and local figures of merit, such as those proposed here, are mandatory. Moreover, the figures of merit introduced here allows to easily obtain quantitative insight; for instance, our results show that when doping is increased (from $10^{16} \mathrm{~cm}^{-3}$ to $10^{17} \mathrm{~cm}^{-3}$ ), for low tip radius the depletion piezopotential is very well preserved, thus resulting in an even increased depletion-to-enhancement piezopotential ratio. This work can great facilitate systematic analyses of axially strained piezosemiconductive nanostructures and can provide insight for the design of better piezoelectric nanotransducers.

Author Contributions: Conceptualization, P.A. and C.F.; software, P.A.; validation, P.A.; data curation, P.A.; writing—original draft preparation, C.F.; writing—review and editing, C.F.; visualization, P.A.; supervision, C.F. All authors have read and agreed to the published version of the manuscript.

Funding: This research received no external funding.

Acknowledgments: The authors thank Franco Di Paolo for support with FEM simulations.

Conflicts of Interest: The authors declare no conflict of interest.

\section{References}

1. Falconi, C. Piezoelectric nanotransducers. Nano Energy 2019, 59, 730-744. [CrossRef]

2. Wang, X.; Zhou, J.; Song, J.; Liu, J.; Xu, N.; Wang, Z.L. Piezoelectric field effect transistor and nanoforce sensor based on a single ZnO nanowire. Nano Lett. 2006, 6, 2768-2772. [CrossRef] [PubMed]

3. Koka, A.; Sodano, H.A. High-sensitivity accelerometer composed of ultra-long vertically aligned barium titanate nanowire arrays. Nat. Commun. 2013, 4. [CrossRef] [PubMed]

4. Nguyen, T.D.; Deshmukh, N.; Nagarah, J.M.; Kramer, T.; Purohit, P.K.; Berry, M.J.; McAlpine, M.C. Piezoelectric nanoribbons for monitoring cellular deformations. Nat. Nanotechnol. 2012, 7, 587-593. [CrossRef] [PubMed]

5. Zhu, G.; Wang, A.C.; Liu, Y.; Zhou, Y.; Wang, Z.L. Functional electrical stimulation by nanogenerator with $58 \mathrm{v}$ output voltage. Nano Lett. 2012, 12, 3086-3090. [CrossRef] [PubMed]

6. Yang, R.; Qin, Y.; Li, C.; Zhu, G.; Wang, Z.L. Converting Biomechanical Energy into Electricity by a Muscle-Movement-Driven Nanogenerator. Nano Lett. 2009, 9, 1201-1205. [CrossRef]

7. Li, Z.; Zhu, G.; Yang, R.; Wang, A.C.; Wang, Z.L. Muscle-driven in vivo nanogenerator. Adv. Mater. 2010, 22, 2534-2537. [CrossRef]

8. Shi, B.; Li, Z.; Fan, Y. Implantable Energy-Harvesting Devices. Adv. Mater. 2018, 30, 1801511. [CrossRef]

9. Zheng, Q.; Shi, B.; Li, Z.; Wang, Z.L. Recent Progress on Piezoelectric and Triboelectric Energy Harvesters in Biomedical Systems. Adv. Sci. 2017, 4, 1-23. [CrossRef]

10. Dagdeviren, C.; Li, Z.; Wang, Z.L. Energy Harvesting from the Animal/Human Body for Self-Powered Electronics. Annu. Rev. Biomed. Eng. 2017, 19, 85-108. [CrossRef]

11. Wang, Z.L. Piezotronic and Piezophototronic Effects. J. Phys. Chem. Lett. 2010, 1, 1388-1393. [CrossRef]

12. Starr, M.B.; Shi, J.; Wang, X. Piezopotential-driven redox reactions at the surface of piezoelectric materials. Angezw. Chem. Int. Ed. 2012, 51, 5962-5966. [CrossRef] [PubMed]

13. Ding, Y.; Ma, C.; Wang, Z.L. Self-Catalysis and Phase Transformation in the Formation of CdSe Nanosaws. Adv. Mater. 2004, 16, 1740-1743. [CrossRef]

14. Xue, X.; Nie, Y.; He, B.; Xing, L.; Zhang, Y.; Wang, Z.L. Surface free-carrier screening effect on the output of a ZnO nanowire nanogenerator and its potential as a self-powered active gas sensor. Nanotechnology 2013, 24, 225501. [CrossRef] [PubMed]

15. Hu, Y.; Klein, B.D.B.; Su, Y.; Niu, S.; Liu, Y.; Wang, Z.L. Temperature dependence of the piezotronic effect in ZnO nanowires. Nano Lett. 2013, 13, 5026-5032. [CrossRef] [PubMed]

16. Xue, F.; Zhang, L.; Tang, W.; Zhang, C.; Du, W.; Wang, Z.L. Piezotronic effect on ZnO nanowire film based temperature sensor. ACS Appl. Mater. Interfaces 2014, 6, 5955-5961. [CrossRef] [PubMed]

17. Falconi, C.; D’Amico, A.; Wang, Z.L. Wireless nanosensors and nanoactuators for in-vivo biomedical applications. In Proceedings of the Eurosensors, Göteborg, Sweden, 17-20 September 2006. 
18. Falconi, C.; D'Amico, A.; Wang, Z.L. Wireless Joule nanoheaters. Sens. Actuators B Chem. 2007, 127, 54-62. [CrossRef]

19. Ciofani, G.; Danti, S.; D’Alessandro, D.; Ricotti, L.; Moscato, S.; Bertoni, G.; Falqui, A.; Berrettini, S.; Petrini, M.; Mattoli, V.; et al. Enhancement of Neurite Outgrowth in Neuronal-Like Cells following Boron Nitride Nanotube-Mediated Stimulation. ACS Nano 2010, 4, 6267-6277. [CrossRef]

20. Wang, A.; Liu, Z.; Hu, M.; Wang, C.; Zhang, X.; Shi, B.; Fan, Y.; Cui, Y.; Li, Z.; Ren, K. Piezoelectric nanofibrous scaffolds as in vivo energy harvesters for modifying fibroblast alignment and proliferation in wound healing. Nano Energy 2018, 43, 63-71. [CrossRef]

21. Falconi, C.; Mantini, G.; D'Amico, A.; Wang, Z.L. Studying piezoelectric nanowires and nanowalls for energy harvesting. Sensors Actuators B Chem. 2009, 139, 511-519. [CrossRef]

22. Araneo, R.; Lovat, G.; Burghignoli, P.; Falconi, C. Piezo-semiconductive quasi-1D nanodevices with or without anti-symmetry. Adv. Mater. 2012, 24, 4719-4724. [CrossRef] [PubMed]

23. Araneo, R.; Falconi, C. Lateral bending of tapered piezo-semiconductive nanostructures for ultra-sensitive mechanical force to voltage conversion. Nanotechnology 2013, 24, 265707. [CrossRef] [PubMed]

24. Hao, H.; Jenkins, K.; Huang, X.; Xu, Y.; Huang, J.; Yang, R. Piezoelectric potential in single-crystalline ZnO nanohelices based on finite element analysis. Nanomaterials 2017, 7, 430. [CrossRef] [PubMed]

25. Han, J.H.; Park, K.I.; Jeong, C.K. Dual-structured flexible piezoelectric film energy harvesters for effectively integrated performance. Sensors 2019, 19, 1444. [CrossRef] [PubMed]

26. Wang, L.; Zhao, L.; Jiang, Z.; Luo, G.; Yang, P.; Han, X.; Li, X.; Maeda, R. High accuracy comsol simulation method of bimorph cantilever for piezoelectric vibration energy harvesting. AIP Adv. 2019, 9. [CrossRef]

27. Wang, Z.L.; Song, J. Piezoelectric nanogenerators based on zinc oxide nanowire arrays. Science 2006, 312, 242-246. [CrossRef] [PubMed]

28. Gao, Y.; Wang, Z.L. Electrostatic potential in a bent piezoelectric nanowire. The fundamental theory of nanogenerator and nanopiezotronics. Nano Lett. 2007, 7, 2499-2505. [CrossRef]

29. Xu, S.; Wang, Z.L. One-dimensional ZnO nanostructures: Solution growth and functional properties. Nano Res. 2011, 4, 1013-1098. [CrossRef]

30. Xia, B.Y.; Yang, P.; Sun, Y.; Wu, Y.; Mayers, B.; Gates, B.; Yin, Y.; Kim, F.; Yan, H.; Xia, Y.; et al. One-Dimensional Nanostructures: Synthesis, Characterization, and Applications. Adv. Mater. 2003, 15, 353-389. [CrossRef]

31. Arrabito, G.; Errico, V.; Zhang, Z.; Han, W.; Falconi, C. Nanotransducers on printed circuit boards by rational design of high-density, long, thin and untapered $\mathrm{ZnO}$ nanowires. Nano Energy 2018, 46, 54-62. [CrossRef]

32. Özgür, Ü.; Alivov, Y.I.; Liu, C.; Teke, A.; Reshchikov, M.A.; Doğan, S.; Avrutin, V.; Cho, S.J.; Morko, H. A comprehensive review of ZnO materials and devices. J. Appl. Phys. 2005, 98, 1-103. [CrossRef]

33. Gao, Y.; Wang, Z.L. Equilibrium Potential of Free Charge Carriers in a Bent Piezoelectric Semiconductive Nanowire. Nano Lett. 2009, 9, 1103-1110. [CrossRef] [PubMed]

34. Romano, G.; Mantini, G.; Di Carlo, A.; D’Amico, A.; Falconi, C.; Wang, Z.L. Piezoelectric potential in vertically aligned nanowires for high output nanogenerators. Nanotechnology 2011, 22, 465401. [CrossRef] [PubMed]

35. Araneo, R.; Lovat, G.; Falconi, C.; Notargiacomo, A.; Rinaldi, A. Accurate analysis of the piezopotential and the stored energies in laterally bent piezo-semiconductive NWs. In Proceedings of the Materials Research Society Symposium Proceedings, Boston, MA, USA, 25-30 November 2012; Volume 1556, pp. 89-94.

36. Araneo, R.; Lovat, G.; Falconi, C.; Notargiacomo, A.; Rinaldi, A. Accurate models for the current-voltage characteristics of vertically compressed piezo-semiconductive quasi-1D NWs. Mater. Res. Soc. Symp. Proc. 2013, 1556, 11-16. [CrossRef]

37. Tao, R.; Mouis, M.; Ardila, G. Unveiling the Influence of Surface Fermi Level Pinning on the Piezoelectric Response of Semiconducting Nanowires. Adv. Electron. Mater. 2018, 4, 1-9. [CrossRef]

38. Min Kim, S.; Kim, H.; Nam, Y.; Kim, S. Effects of external surface charges on the enhanced piezoelectric potential of ZnO and AlN nanowires and nanotubes. AIP Adv. 2012, 2, 42174. [CrossRef]

39. Fathi, S.; Sheikholeslami, T.F. Investigation of external charges effects on piezoelectric ZnO nanogenerator. J. Nano Electron. Phys. 2016, 8, 6-11. [CrossRef] 